\title{
A Numerical Simulation of a Vortex Convected Through a Laminar Premixed Flame
}

\author{
MING-SHIN WU and JAMES F. DRISCOLL \\ Department of Aerospace Engineering, University of Michigan, Ann Arbor MI 48109
}

\begin{abstract}
A numerical study was conducted to understand how a vortex, when convected at moderate speeds across a premixed flame, can induce velocities that pull the flame along with the vortex, causing flame elongation and unsteady flame stretch. If the vortex-induced velocity that opposes flame motion is sufficiently large, the flame cannot propagate over the vortex and thus temporarily remains attached to the moving vortex. A flame attachment criterion is discussed; when the criterion is met the vortex forms cusps and pockets in the flame structure similar to those observed experimentally. The net result of increasing the vortex convection velocity is to reduce the residence time of the vortex in the flame, which reduces the degree of flame wrinkling. Vortex pairs that exert an extensive strain on the flame were found to have significantly longer residence times of interaction than vortices that exert compressive strain; this difference in residence time helps to explain why extensive strain on a flame is more probable in turbulent flames than compressive strain. The calculated images of the laminar flame shape show encouraging agreement with experiment, which is another indication that flame-interface simulations are a promising way to represent very wrinkled turbulent premixed flames in a numerically efficient manner.
\end{abstract}

\section{INTRODUCTION}

Previous simulations of an interaction between a laminar vortex and a premixed flame have concentrated on cases for which there is no convection velocity of the vortex towards the flame [1-5] or for which the self-induced convection velocity of the vortex is relatively small and is not varied [6-8]. Some of the previous simulations have predicted that the vortex will have a large residence time in the flame, causing the flame to roll up and form as many as four spiral-shaped layers as the vortex rotates. These predicted flames do not appear to realistically represent turbulent flames because such spiral-shaped flames have not been observed experimentally. In most turbulent flames each vortex has a convection velocity that limits the residence time of the vortex in the flame so that flame rollup is limited to less than one revolution. For example, turbulent eddies pass through rod-stabilized flames with large convective velocities relative to their rotational velocities. The present work adds moderate convection velocities to the simplest case of a laminar flame-vortex interaction and shows that the resulting flame shapes do realistically represent the experimental observations of Roberts and Driscoll [9].

A flame-interface simulation was used rather than a full Navier-Stokes simulation. The interface simulation represents the flame as an infinitely thin interface and therefore makes no attempt to resolve the structure within the flamefront; instead a propagation speed must be assigned to each part on the interface. Such a technique requires enormously less computer memory and therefore is a promising method to represent very wrinkled turbulent flames at high Reynolds number. The simple line interface calculation (SLIC) that was used in the present work has been used to represent premixed flames within other types of flowfields, as reported by Chorin [1], Ashurst and Barr [10], Ghoniem and Knio [11], $\mathrm{Wu}$ et al. [12], and Kwon et al. [13]. The latter two simulations of very wrinkled turbulent flames predicted trends for the flame perimeter and wrinkle length scale that are in agreement with experiment. Another type of flame-interface simulation that differs from the SLIC algorithm used herein is generated by solving a 
field equation that is denoted the $\mathrm{G}$ equation, as reported by Kerstein et al. [14], Ashurst et al. [15], and others. Osher and Sethian [16] note that both the $G$ equation solutions and SLIC computation that is used herein represent the same general approach, although the algorithms differ.

To implement a flame-interface simulation, the velocity field first is specified. For turbulent flow cases (i.e., not the present case) a discrete vortex method or a stochastic representation $[10,12,13]$ can be used. For laminar flows, such as the present case, a sinusoidal or a vortex velocity field has been used $[1,3]$. The flame is represented by a thin interface that represents many practical laminar and turbulent situations. The interface is propagated normal to itself either by using SLIC, or by computing isoclines of the $\mathrm{G}$ equation [14-16]. The advantage of interface simulations over statistical pdf methods to represent turbulent flames is that the interface method yields an instantaneous image of the wrinkled flame. Such images are felt to be essential in order to assess the physics of the simulation, to add local stretch effects, and to provide comparison to experiment.

In the present work, counterrotating vortex pairs were considered that exert either extensive or compressive strain on the flames, as shown in Fig. 1a. The residence times of the interaction for the two cases were compared in order to determine if one case is more probable in a turbulent flow. Figure 1a show that two adjacent vortices can be (a) co-rotating (not shown) or (b) counterrotating, such that reactants between the vortices are directed away from (against) the flame for the compressive strain case (extensive strain case). The authors believe that a major reason that extensive strain is more probable than compressive strain is because the flame propagates rapidly over a vortex pair exerting compressive strain since the reactants between the vortices are moving in the direction of flame propagation, as shown in Fig. 1a. A much longer residence time of interaction is expected to occur when a vortex pair exerts extensive strain because the flowfield between vortices is directed against the flame propagation. The present results verify this physical reasoning.

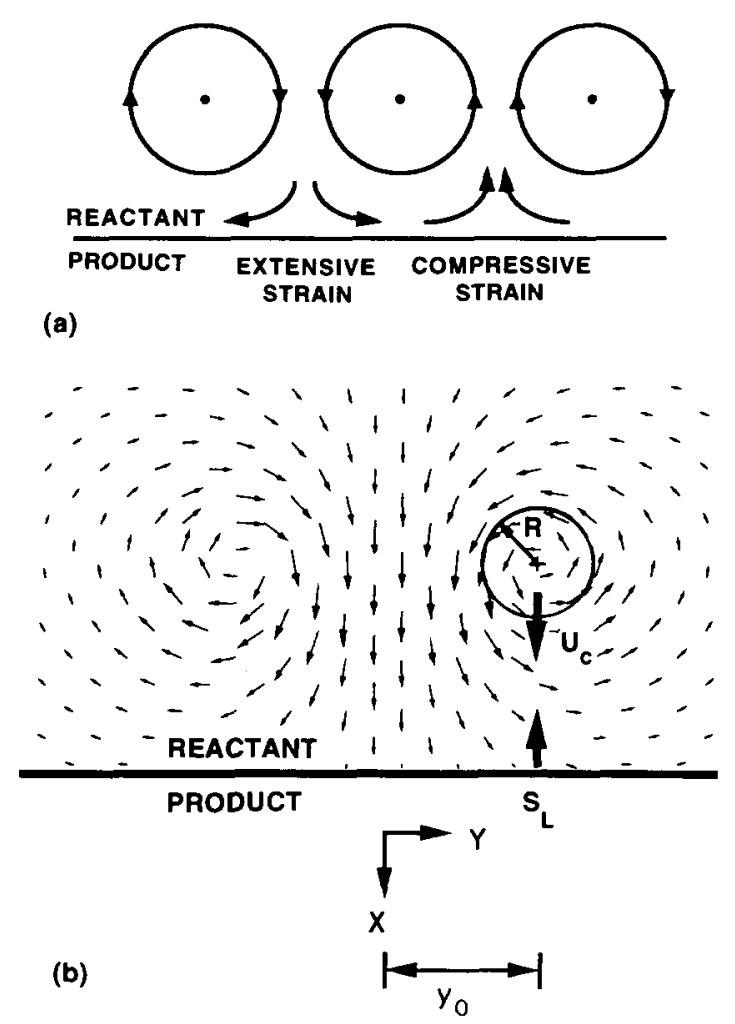

Fig. 1. Schematic of (a) adjacent counterrotating vortices in a turbulent flow and (b) the Oseen vortex pair considered in the present work, with vectors drawn to scale.

\section{THE NUMERICAL ALGORITHM}

A pair of two-dimensional Oseen vortices were interacted with a laminar premixed flame, as is shown in Fig. 1b. The assumptions made in the simulation are (a) the velocity field is frozen in time, (b) the local flame propagation speed is everywhere constant (no stretch), (c) thermal expansion is neglected, (d) the buoyancy forces also are zero, so the baroclinic torque is neglected, and (e) the flow field is two dimensional. Each of the above assumptions could be relaxed in the future in order to improve the quantitative agreement with experiment. For example, flame stretch was not included in the present work; it is possible to include flame stretch in the future by computing the interface curvature and local strain rate and then appropriately modifying the local burning velocity $S_{L}$. Heat release causes gas expansion, which distorts the velocity field; gas expansion effects can be included by adding a distribution 
of sources of fluid on the interface [11]. The Oseen vortex pair was chosen because such a velocity field realistically represents the velocities measured in the cross-sectional plane of a toroidal vortex that was generated experimentally [9] and because the effects of viscous decay of the vortex can be represented. Some calculations also were done using a single vortex, as described below. For a single Oseen vortex, the velocity vector is oriented in the azimuthal direction and has a magnitude [V] given by [17]

$$
[V]=(\Gamma /(2 \pi r))\left(1-\exp \left(-r^{2} / R^{2}\right)\right),
$$

where $R$ is the vortex core radius and $\Gamma$ is the vortex circulation. For a single Oseen vortex, the maximum azimuthal velocity is 0.638 $\Gamma /(2 \pi R)$ and this maximum azimuthal velocity occurs at a radial location of $1.12 R$. Viscous decay of an Oseen vortex can be represented by allowing the core radius $R$ to vary with time and viscosity [17]; in the present work $R$ was held constant. The Oseen vortex pair satisfies the continuity equation.

The three parameters that govern the interaction between the flame and the vortex pair shown in Fig. $1 \mathrm{~b}$ are $U_{\theta} / S_{L}, U_{C} / U_{\theta}$, and $R / y_{0}$. The vortex core radius is $R$ and the distance between the vortex centers is $2 y_{0} . U_{\theta}$ is the characteristic rotational velocity and is defined as the maximum velocity induced by the vortex pair in the $y$ direction, which is the transverse direction shown in Fig. 1b. $S_{L}$ is the laminar burning velocity; $U_{C}$ is the convection velocity of the vortex centers with respect to the unburned gas that is ahead of the flame when the flame is at upstream infinity. Therefore the relative velocity between the flame and the vortex centers is $\left(S_{L}+U_{C}\right)$ before interaction begins. All values of time are normalized by the characteristic transit time which equals $2 y_{0} /\left(S_{L}+U_{C}\right)$.

It is important to include the convective velocity $U_{C}$ as a parameter because this velocity has been shown to be important in realistic turbulent flames $[12,13]$ and in flame-vortex interaction experiments [9]. In a turbulent flow, a vortex will have a convective velocity that is induced by all neighboring vortices. A convective velocity also can result from the mean flow field, for example, when turbulent vortices pass through a $\mathrm{V}$-shaped flame. Values that were selected for the governing parameters were $U_{\theta} / S_{L}=1,10$, and $50, R / y_{0}=0.08,0.2$, and 0.4 , and $U_{C} / U_{\theta}=0.0$ to 1.1 . These values overlap those of the experiment [9] for which $U_{\theta} / S_{L}$ was 1 to $50, R / y_{0}=0.08$, and $U_{C} / U_{\theta}=1.0$.

The flame interface was simulated using the simple line interface calculation (SLIC) method developed by Noh and Woodward [18] and by Chorin [1] and is used in Refs. 1, 10-13, and 19. Initially a horizontal flame is located at a distance of $2 y_{0}$ below the vortex centers. The initial location of the flame was varied from $2 y_{0}$ to $8 y_{0}$ but there was no substantial difference in the results. The computational domain is a 200 by 200 grid of dimensions $4 y_{0}$ by $4 y_{0}$; the grid spacing is $0.02 y_{0}$. The SLIC algorithm first advects the interface during each time step using the known velocity field of the Oseen vortex pair. To each volume element formed by the grid, a value is assigned between zero and one that represents the fraction of the volume occupied by products. The remaining volume fraction represents reactants. The SLIC algorithm represents the interface only as horizontal lines, vertical lines or corners within each volume element. These horizontal and vertical lines are efficiently moved in the $x$ and $y$ directions as specified by the two velocity components and a new volume fraction of products is computed. The flame interface is defined as the contour along which the volume fraction of products equals 0.5 .

The algorithm next propagates the interface normal to itself at the local burning velocity $S_{L}$. In general, $S_{L}$ can depend on flame stretch, since flame curvature and local strain can be computed and can be related to $S_{L}$ if an empirical or theoretical relation for $S_{L}$ is used. In the present work $S_{L}$ was held constant. To propagate the flame normal to itself, Huyghen's principle is used, which states that every point on the interface acts like a point source of a spherical wave. At each interface location eight test cases are executed during each time step; each case represents wave propagation at a different direction to the interface. The case that yields a maximum volume of products represents propagation that is normal to the interface. Finally, during each time step the 
vortex convection velocity $\left(U_{C}\right)$ is simulated by advancing the location of the vortex centers.

The time increment was set to be less than $\Delta x /\left(2 U_{x, \max }\right)$ in order to satisfy the Courant condition, where $\Delta x$ is the grid spacing and $U_{x, \max }$ is the maximum velocity in the $x$ direction. A typical run time to execute 2000 time steps on a DEC 5000 computer was 5 hours. Much shorter run times are possible if the algorithm is optimized to store only information concerning the interface location rather than the entire grid. The 200 by 200 grid was determined to be sufficiently fine; the grid spacing was reduced by a factor of 2 and the general flame geometry did not change while the calculated flame perimeter increased only by ten percent.

\section{CRITERION FOR THE FLAME TO ATTACH TO THE VORTEX}

Consider the vortex pair shown in Fig. 1b; a laboratory coordinate system is defined in which the unburned reactants initially are at rest. $U_{x, c l}$ is defined as the $x$ component of the velocity induced by the vortex pair on the centerline $(y=0)$, which varies in space as shown. Now this vorticity field is convected downward at $U_{C}$ in the laboratory frame. Only the vorticity field is convected, not the fluid elements in the vortex, so the induced velocity at a fixed point on the centerline still is $U_{x, \mathrm{cl}}$. Now consider a coordinate system attached to the center of one vortex. A fluid element just ahead of the flame on the centerline has velocity $\left(U_{x, \mathrm{cl}}-U_{C}\right)$ in this vortex frame of reference and the flame will have velocity $S_{L}$ with respect to this fluid element. Therefore the flame/vortex relative velocity is $S_{L}-\left(U_{x, \mathrm{cl}}-\right.$ $\left.U_{C}\right)$. This relation shows that prior to the interaction, when $U_{x, \text { cl }}$ is zero, the flame/vortex relative velocity is $\left(S_{L}+U_{C}\right)$. As the vortex approaches the flame, $U_{x, \mathrm{cl}}$ increases until the flame/vortex relative velocity becomes zero and the flame appears to be attached to the vortex on the centerline. Therefore flame attachment will occur on the centerline if the vortex pair has sufficient rotational velocity so that

$U_{x, \mathrm{cl}, \max }>S_{L}+U_{C}$.
$U_{x, \mathrm{cl}, \max }$ is calculated for a given vortex or vortex pair by using Eq. 1 and the resulting values are given in the next section.

\section{RESULTS-SIMULATION OF FLAME ROLLUP AND POCKET FORMATION}

Figure 2 shows a typical flame-vortex interaction. The crosses indicate the vortex centers and the circles that surround the crosses are vortex core boundaries, where the rotational velocity is near maximum. The solid line indicates the flame position.

It is observed in Fig. 2a that as the vortex is convected downward through the flame, the flame on the centerline $(y=0)$ is forced to move downward with the vortex. The flame attachment criterion (Eq. 2) is met in this case since $U_{x, \text { cl, } \max }$ is $0.64 U_{\theta}, U_{C}$ is $0.16 U_{\theta}$, and $S_{L}$ is $0.1 U_{\theta}$. Note that the flame at the leading edge of the vortex never burns upwards over the vortex core; it remains nearly fixed with respect to the vortex centers. The pocket of reactants is consumed because the flame at the rear of the vortex eventually burns downwards over the vortex. The net result of flame attachment is that the flame surface is greatly elongated (stretched) in an unsteady manner. Figure $2 \mathrm{a}$ also shows that two small pockets of reactants near the vortex core are convected downward with the vortex since the attachment criterion also is met in this region. A cusp is observed to form in the primary flame, and this cusp points towards the products, which is consistent with observations of cusps within turbulent flames $[20,21]$. The cusp formation is due to Huyghen's principle; Ashurst et al. [15] have shown that any flame wrinkle will develop into a cusp shape that points to the products because the flame propagates normal to itself.

The simulations of Fig. $2 b$ look similar to the measured flame contours obtained from experiment [9], which are shown in Fig. 2c. No attempt was made to match all the governing parameters of the simulation to the experiment since flame stretch and gas expansion were not simulated but could be added in a straightforward manner in the future. The interface simulation reproduces much of the physics of the experiment, including flame attachment, flame rollup, burn-through from the 


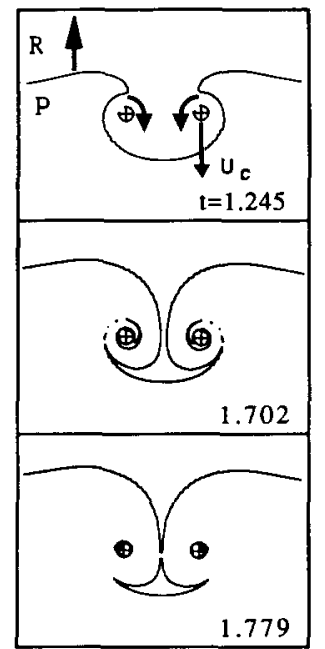

(a) SIMULATION

$$
\begin{aligned}
& U_{\theta} / S_{L}=10 \\
& U_{C} U_{\theta}=0.16 \\
& R / y_{0}=0.2
\end{aligned}
$$

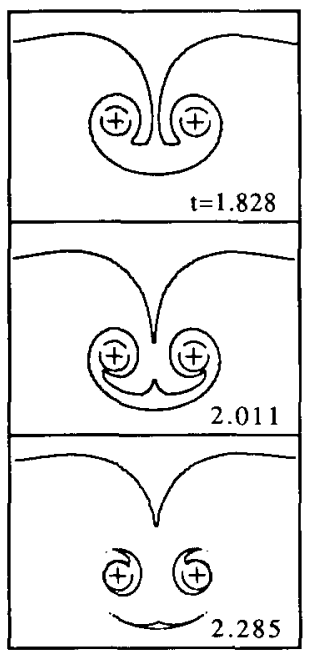

(b) SIMULATION

$U_{\theta} / S_{L}=10$

$U_{C} U_{\theta}=0.34$

$\mathrm{R} / \mathrm{y}_{0}=0.4$

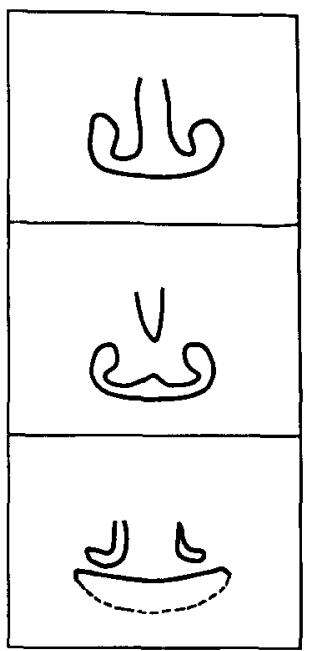

(c) EXPERIMENT

$U_{\theta} / S_{L}=10.2$

$U_{C} / U_{\theta}=1.0$

$R / y_{0}=0.08$
Fig. 2. Comparison of general trends displayed by the simulations and the experiment of Ref. 9. All three governing parameters are not matched since only qualitative comparison is made. Time $t$ is zero when the undisturbed flame is $2 y_{0}$ away from the vortex centers. Time is normalized by $2 y_{0} /\left(U_{C}\right.$ $\left.+S_{L}\right)$. rear of the vortex, and cusp and pocket formation. It is noted that there are no adjustable parameters in the algorithm used; even the absolute values of laminar burning velocity is not specified; instead the ratio $U_{\theta} / S_{L}$ is specified. The entire input consists of values of $U_{\theta} / S_{L}, U_{C} / U_{\theta}, R / y_{0}$, and the velocity field of the form given by Eq. 1 .

The flame-interface simulations shown in Fig. 2 yield a flame shape that is very similar to full Navier-Stokes direct simulations of Poinsot et al. [7, 8]. Poinsot et al. show that the flame attaches to the vortex, the neck region burns through to form a cusp, and a pocket of reactants form, all of which are shown in Fig. 2. For their condition $\left(U_{C}=0, U_{\theta} / S_{L}=28\right)$ the attachment criteria given by Eq. 2 is met.

\section{EFFECTS OF VARYING THE VORTEX CONVECTION VELOCITY}

Some effects of varying the convection velocity are shown in Figs. 3-5 for a constant vortex core radius $R / y_{0}$ of 0.08 . Figures $3-5$ verify that flame attachment occurs when the criterion of Eq. 2 is met. Equation 2 is satisfied in Figs. $3 b, 3 c$, and 4 and it is seen that the flame on the centerline is pulled along with the vortex. Equation 2 is not satisfied in Figs. $3 a$ and 5 and it is seen that the flame does not attach to the vortex but simply propagates over the vortex. Values of $U_{x, \mathrm{cl}, \max }$ are listed in the figure captions. The circular-shaped flame regions that surround the two vortex cores also move downwards with the vortex, as seen in Figs. $3 b$, $3 c, 4 a, 4 b, 5 b$, and $5 c$ since the attachment criterion is met in these regions.

As expected, a stronger vortex (Fig. 3c) causes a greater degree of flame rollup than a weaker vortex (Fig. 3a). Larger convection velocities, on the other hand, provide less residence time for interaction and yield less rollup. Figures $3 c, 4 b$, and $5 c$ all have the same vortex strength $\left(U_{\theta} / S_{L}\right)$ but have correspondingly larger convection velocities $\left(U_{C} / U_{\theta}\right)$; it is seen that the largest convection velocity (Fig. 5c) causes significantly less flame rollup than in Fig. $3 \mathrm{c}$; thus convection velocity has a large effect on the flame shape.

The lateral distance over which the flame is wrinkled is confined to a smaller region when the convection velocity is large. The flame in the middle image of Fig. $3 c$ is disturbed over a lateral $(y)$ distance that exceeds 2.5 vortex 

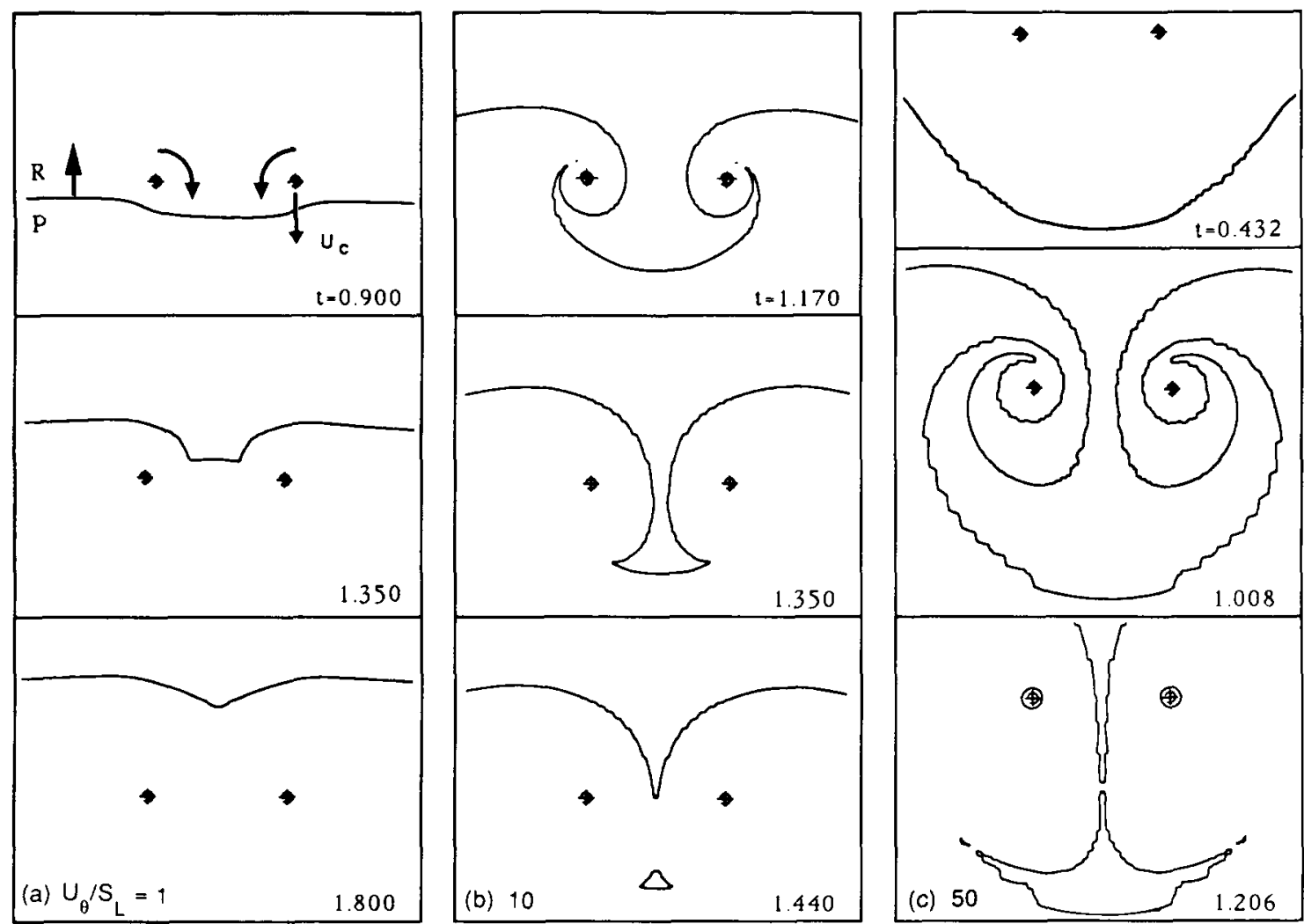

Fig. 3. Effect of increasing vortex strength $\left(U_{\theta} / S_{L}\right)$ on flame rollup for zero convection velocity $\left(U_{C}=0.0\right) . R / y_{0}$ is 0.08 and $U_{x, \max , \mathrm{cl}} / U_{\theta}$ is 0.25 . Time is defined in Fig. 2 .

center spacings, and yet when the convection velocity is large, the flame in Fig. $5 \mathrm{c}$ is disturbed over a distance of only half this amount.

Of particular interest is Fig. 4c, for which the laminar burning velocity is zero. This case might represent a portion of a weak flame in a stratified mixture, or a portion of a stretched flamelet for which $S_{L}$ approaches zero. By comparing Figs. $4 \mathrm{~b}$ and $4 \mathrm{c}$, it is observed that when $S_{L}$ is zero a larger pocket of unburned reactant is formed and no flame rollup occurs. The pocket of reactant is larger in Fig. 4c because the interface is not propagating inward. There is no rollup of the interface in Fig. $4 c$ because the interface always remains far from the vortex centers and so never experiences large rotational velocities. The separate pocket that appears in Fig. $4 \mathrm{c}$ is always connected to the main flame by two vertical flames along the centerline, since the neck region cannot completely burn through in this nonre- acting case. The image of nonreacting interface shown in Fig. $4 \mathrm{c}$ is similar to the direct numerical simulation presented by Laverdant and Candel [6].

It can be concluded from Figs. 2-5 that there are five physical processes that change the flame area: (a) elongation of the wrinkle into a neck-like region due to the convection velocity of the vortex and flame attachment to the vortex, (b) rollup of the flame due to rotational velocities, (c) burn-through of the rolled up layers, (d) burn-through of the neck, creating a pocket and a cusp, and (e) burn-through of the pocket, which sometimes forms smaller pockets. A useful way to show when these physical processes occur is to plot the time history of the computed flame perimeter $\left(P_{T}\right)$, as shown in Fig. 6. The actual flame perimeter $\left(P_{T}\right)$ is normalized by the perimeter of an undisturbed flame $\left(P_{L}\right)$ in Fig. 6; in the present work $P_{L}$ is proportional to the horizontal width 

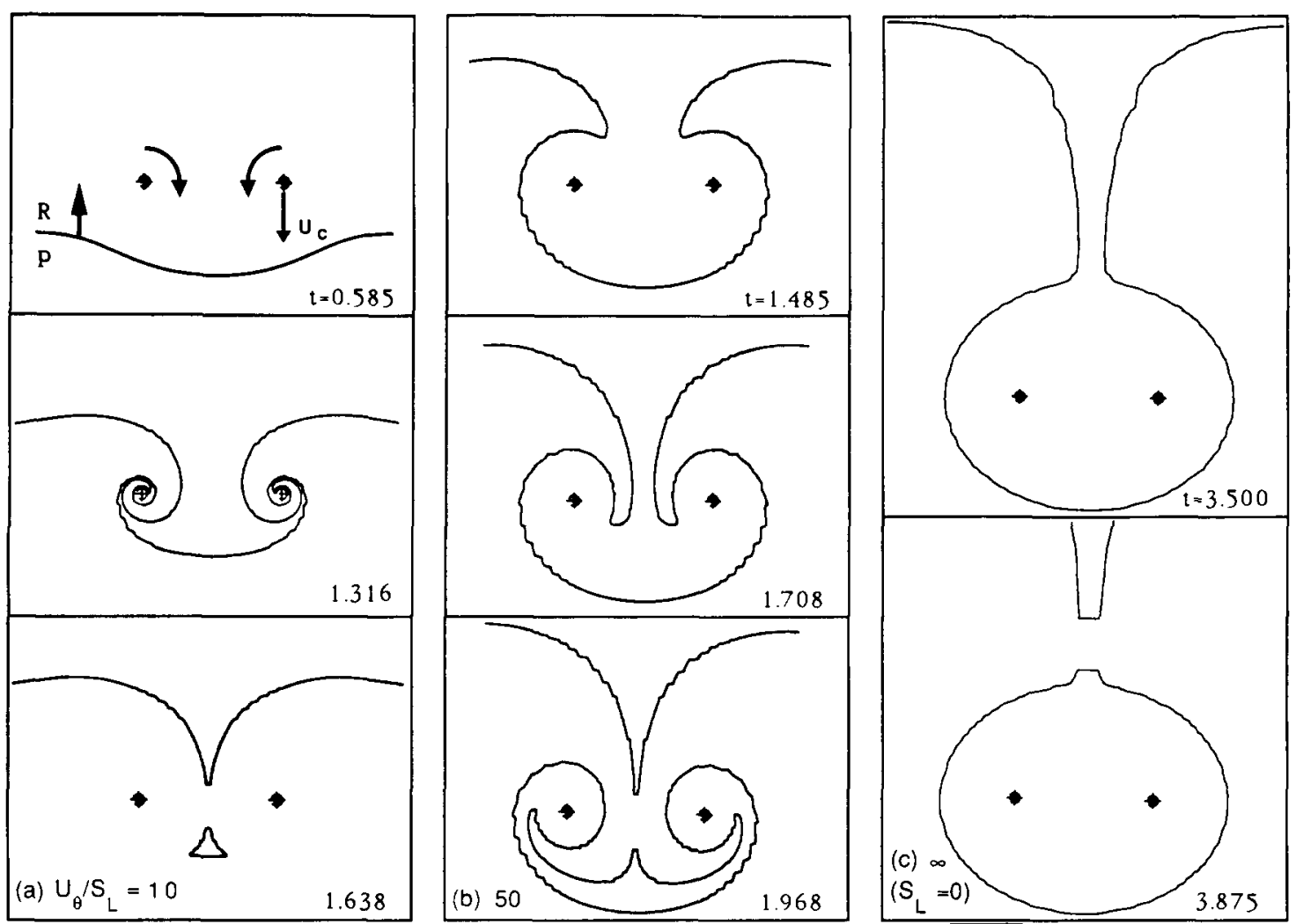

Fig. 4. Flame rollup for medium convection velocity $\left(U_{C} / U_{\theta}=0.063\right) . R / y_{0}$ is 0.08 and $U_{x, \text { max, cl }} / U_{\theta}$ is 0.25 . Time is defined in Fig. 2.

of the computational domain. All five physical processes listed above can be identified by regions of different slope on the curve denoted $U_{\theta} / S_{L}=10$ in Fig. 6 . The nonreacting interface $\left(S_{L}=0\right)$ has a constantly increasing flame perimeter because only the elongation of the wrinkle occurs as was seen in Fig. 4c. Figure 6 also shows that the maximum values of normalized flame perimeter increases from 1.4 to 5 to 9 as the vortex strength is varied from 1 to 10 to 50 , respectively.

The net effect of increasing the vortex convection velocity is to reduce the flame wrinkling, as shown in Fig. 7. The vortex motion across the flame causes two competing effects: the flame is elongated if attachment occurs but the residence time of the interaction also is decreased. The latter effect is what causes the negative slope in Fig. 7. Thus, convection velocity is an important parameter that needs to be correctly simulated to predict a realistic area of a turbulent flame.

\section{REGIME DIAGRAM SHOWING WHEN INTENSE WRINKLING AND POCKET FORMATION OCCUR}

Some of the different regimes of the flame-vortex interaction are plotted in Fig. 8. It is seen that if the vortex is made stronger, one proceeds upwards along a vertical line in Fig. 8 and the wrinkling increases until a pocket is formed. Intense wrinkling is defined to occur when the maximum normalized flame perimeter exceeds four. An unburned region is defined as a small pocket when its area exceeds $0.19 \pi y_{0}^{2}$. The main conclusion drawn from Fig. 8 is that pockets form only for a certain range of convection velocities. The pocket regime boundary is seen to follow a general parabolic trend so that along the horizontal line $U_{\theta} / S_{L}=20$, for example, pockets do not form for sufficiently small or for sufficiently large convection velocities. For sufficiently small convection velocity there is more resi- 

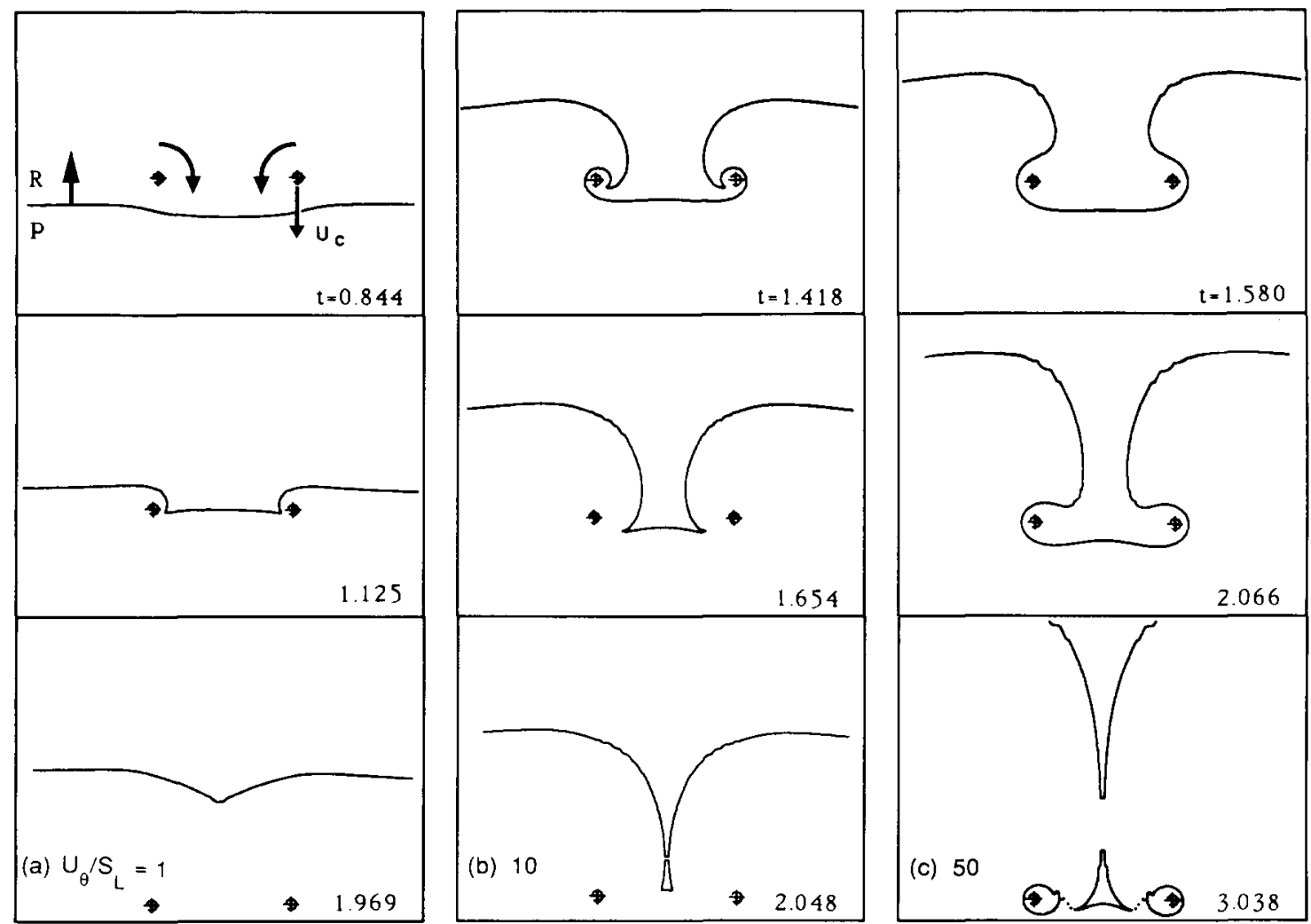

Fig. 5. Flame rollup for large convection velocity $\left(U_{C} / U_{\theta}=0.25\right) . R / y_{0}$ is 0.08 and $U_{x \text {, max. cl }} / U_{\theta}$ is 0.25 . Time is defined in Fig. 2.

dence time for the flame that surrounds the pocket to burn inward, resulting in smaller (or no) pockets. For sufficiently large convection velocity, the flame attachment criterion holds only very near the centerline since large $U_{C}$ increases the right hand side of Eq. 2. Thus only a small region of the flame near the centerline is pulled along with the moving vortex, as seen in Fig. 5b, and no pocket forms.

\section{SINGLE VORTEX AND COUNTERROTATING CASES}

Another case that was considered was that of a single Oseen vortex convected through a flame, which is shown in Fig. 9. The flame initially was located at $25 R$ and the time is normalized by $25 R /\left(S_{L}+U_{C}\right)$, where $R$ is the core radius. As with the vortex pair, Fig. 9a shows that when the attachment criterion described above is not satisfied, the flame does not attach to the vortex and little wrinkling occurs. In Figs.
$9 \mathrm{~b}$ and $9 \mathrm{c}$ the attachment criterion is met and as the vortex is convected downward, the flame attaches and is pulled with the vortex. The effect of varying the convection velocity of the single vortex is observed by comparing Figs. $9 \mathrm{~b}$ and 9c. Larger convection velocity (Fig. 9c) results in less flame rollup because of the reduced residence time, as demonstrated by Fig. 7.

The single vortex cases were found to display all five of the above physical processes that were observed for the vortex pair, namely flame attachment and elongation, flame rollup, and burn-through of the layers and pocket. The flame perimeter $P_{T} / P_{L}$, which was defined previously to be the perimeter of the wrinkled flame (including pocket) normalized by the perimeter of the undisturbed flame, calculated for the single vortex case and is found to be nearly identical to that calculated using the direct numerical simulation of Rutland et al. [4]. Rutland et al. obtained a value of $P_{T} / P_{L}$ 


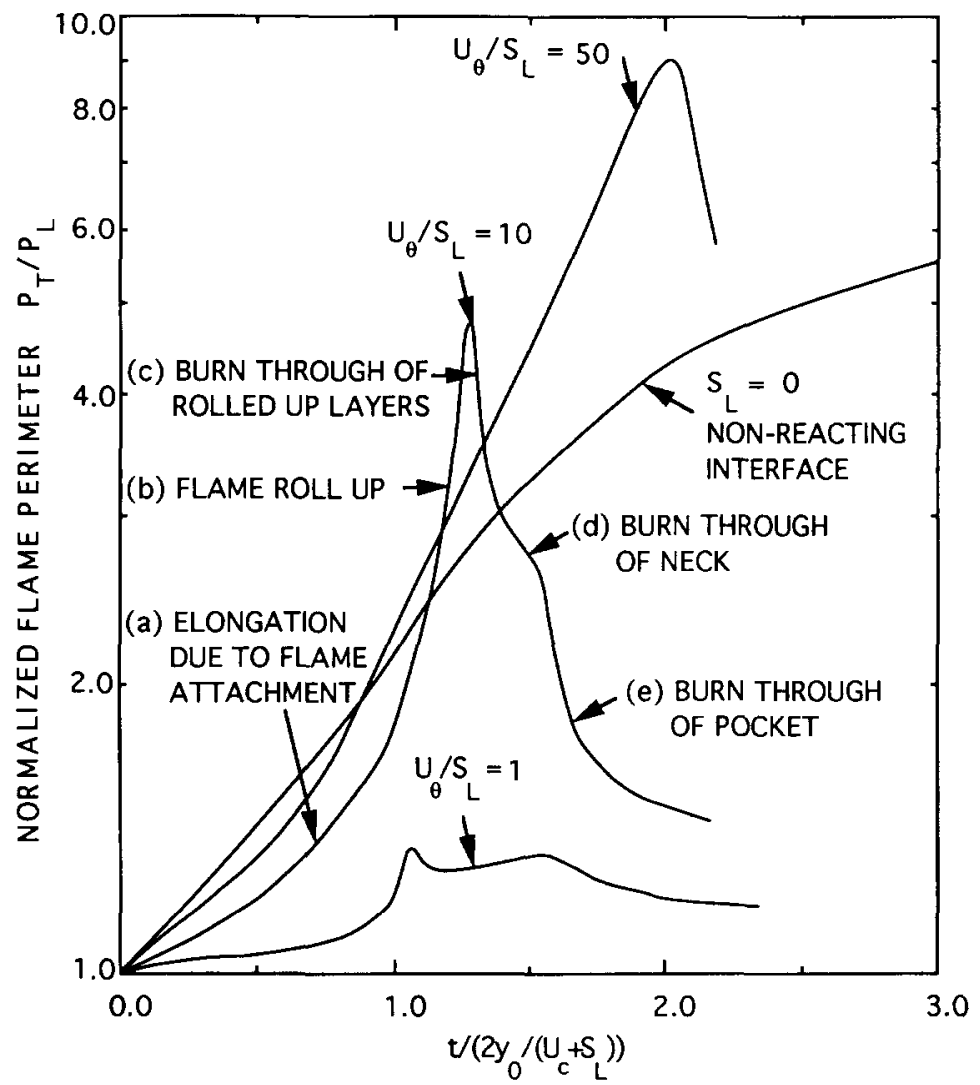

Fig. 6. Time history of flame perimeter which shows when five physical processes occur. Conditions same as Fig. 4. $U_{C} / U_{\theta}=0.063$. Note that the flame perimeter is an indicator of the reactant consumption rate under the assumption of the present simulation. of 1.3 for $U_{\theta} / S_{L}=1.5$ and $U_{C}=0$; the present interface simulation yielded a value of $P_{T} / P_{L}$ of 1.5 for essentially the same conditions.

The degree of flame wrinkling produced by a single vortex is quantified in Fig. 10. The amplitude (Amp) is defined as the maximum distance between the uppermost and lowermost points on the flame in any one image such as in Fig. 9. The wavelength of the disturbance $\lambda_{\mathrm{Amp} / 4}$ is defined as the horizontal distance in Fig. 9 between locations where the flame $x$ location deviates from its undisturbed position by $\mathrm{Amp} / 4$. Figure 10 shows that the wrinkle amplitude can be 10-60 times larger than the vortex core radius $R$ and that the wavelength $\lambda_{\mathrm{Amp} / 4}$ can be even larger than the amplitude. For the strong vortices considered in Fig. 10, the rotational velocities exceed $S_{L}$ over distances of 20 to $80 R$.

Another case that was considered was a vortex pair for which the rotational velocity is opposite to that of Figs. 1-5. The vortex pair shown in Fig. 11 exerts compressive strain on the flame and the velocity on the centerline is in the direction of flame propagation. The interaction for the compressive strain case shown in Fig. 11 results in less residence time than the extensive strain cases shown in Figures $1-5$. For example, Figs. $5 b$ and $11 \mathrm{c}$ represent the same conditions except that the direction of vortex rotation is reversed. We can define the residence time to begin when the undisturbed flame is $2 y_{0}$ away from the vortex centers initially. The residence time is defined to end when the flame passes the vortex centers, as shown in the lowest images in Figure $5 b$ and 11c. Therefore, the nondimensional residence time for the extensive strain case (Fig. 5b) is 2.048 , which is approximately 1.4 time as large as the residence time of the compressive strain case shown in Fig. 11c. The fact that the flame passage is retarded in the extensive strain flowfield (and accelerated in the compressive strain field) is believed to be one reason that extensive strain on the flame is found to be more probable in turbulent flame simulations $[22,23]$.

Figure 11 also demonstrate that the flame 


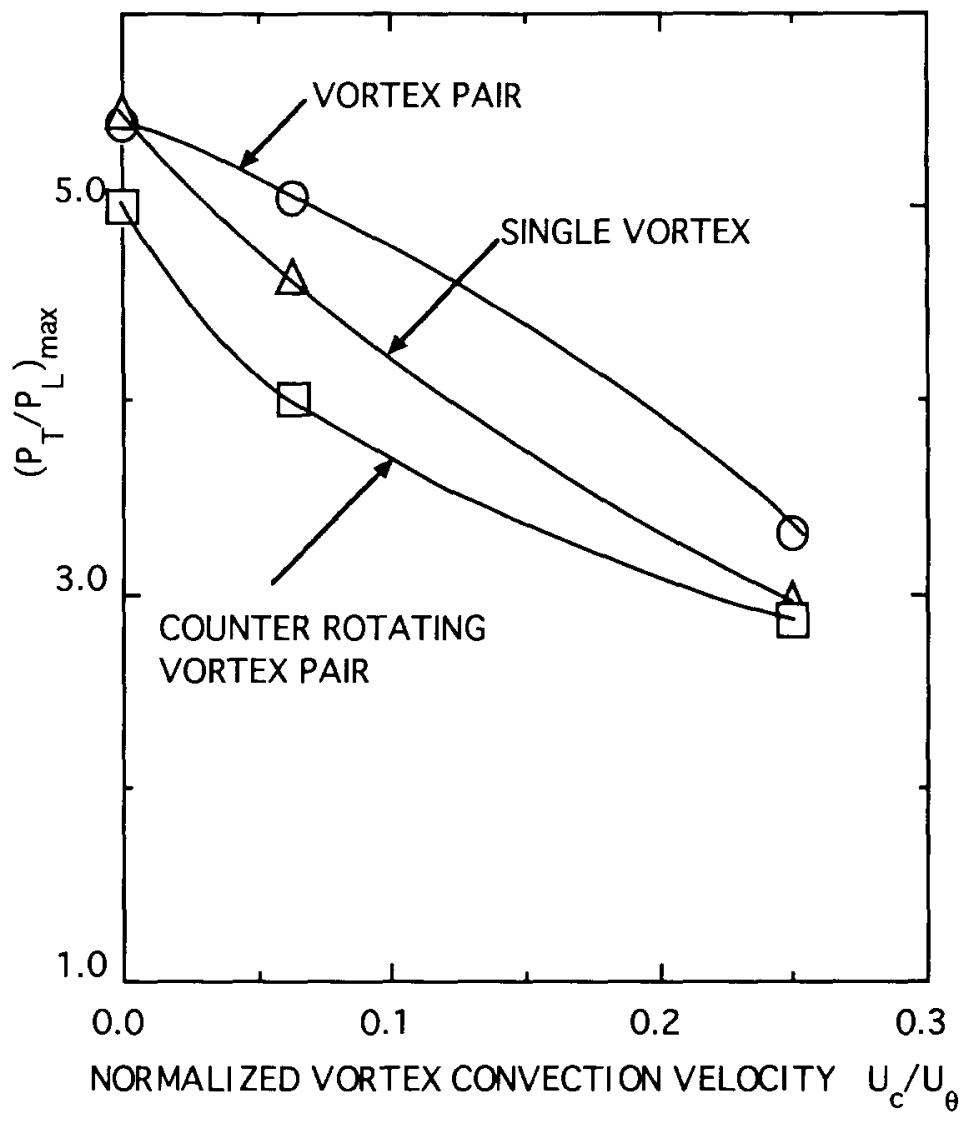

Fig. 7. Net reduction of maximum flame perimeter due to convection velocity of vortex. For all cases $U_{\theta} / S_{L}$ is 10 and $\left(P_{T} / P_{L}\right)_{\max }$ is the maximum of curves shown in Fig. 6. under compressive strain field has less flame rollup, a smaller lateral distance over which the flame is wrinkled, and smaller pockets of reactants than for the flame under extensive strain field shown in Figs. 1-5. The main reason for these differences is that the flame attachment criterion $\left(U_{\theta}>S_{L}+U_{C}\right)$ no longer is met on the centerline, so the flame quickly burns upwards over the vortex cores. As the convection velocity of the vortex in Fig. 11 is increased, the degree of flame wrinkling is decreased, as demonstrated by Fig. 7 .

\section{CONCLUSIONS}

1. It was shown that a flame-interface simulation reproduces the five physical processes that are experimentally observed during a flame-vortex interaction, namely flame attachment to a moving vortex and elongation, flame rollup, and burn-through of layers, a neck region, and a pocket of reac- tants. For better quantitative agreement, it is necessary to allow for flame stretch and gas expansion, which can be done in a straightforward manner. Because it is so computationally efficient, the interface simulation method appears to be a useful way to eventually simulate highly wrinkled turbulent premixed flames.

2. The convection velocity of each vortex through the flame is an important parameter that is shown to alter the flame shape significantly. The convection velocity elongates certain regions of the flame and yet it reduces the residence time and hence the rollup of other regions, resulting in a net decrease in the flame perimeter.

3. A flame attachment criterion is discussed; if the criterion is met, the flame is forced to be advected along with the vortex, which subjects the flame to a large unsteady stretch rates and can result in pockets of unburned reactants. 


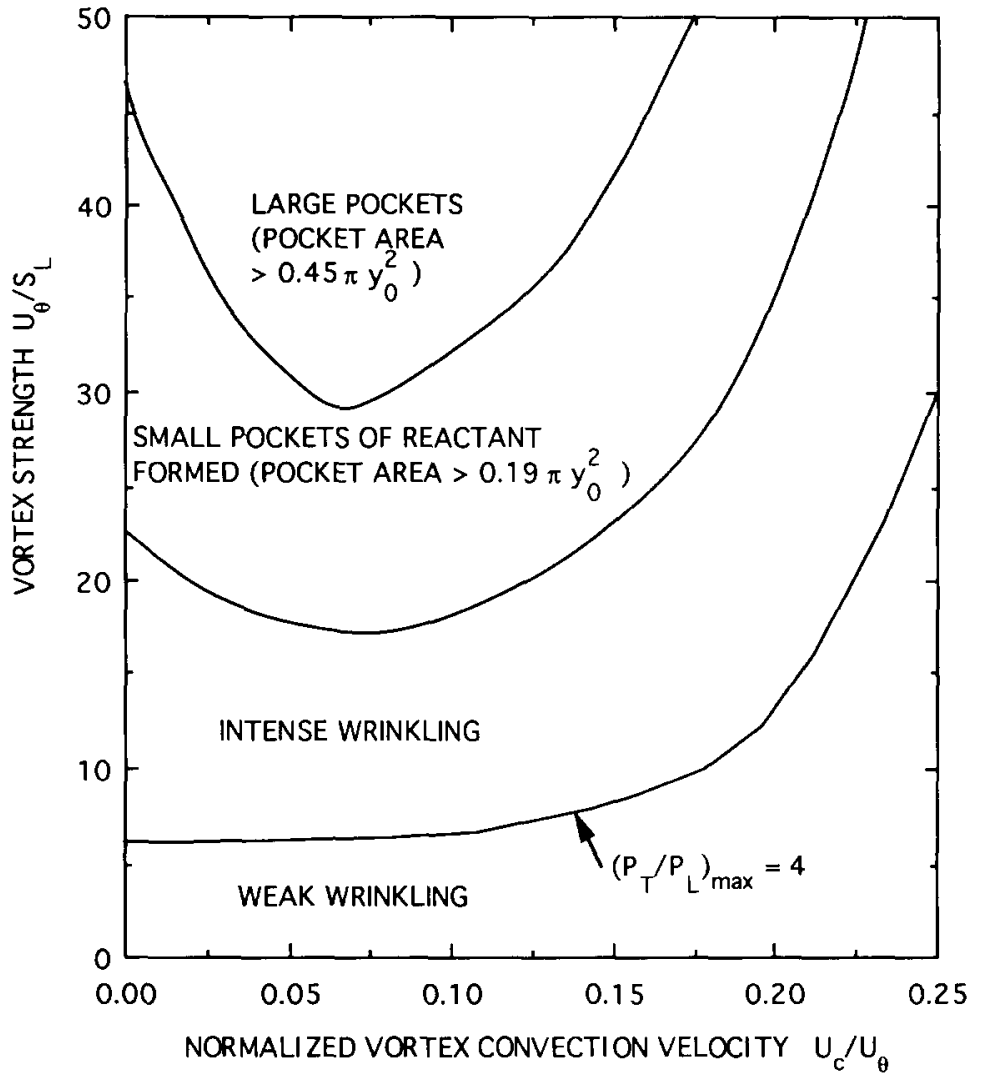

Fig. 8. Regimes of the flame-vortex interaction. $R / y_{0}$ is 0.08 .
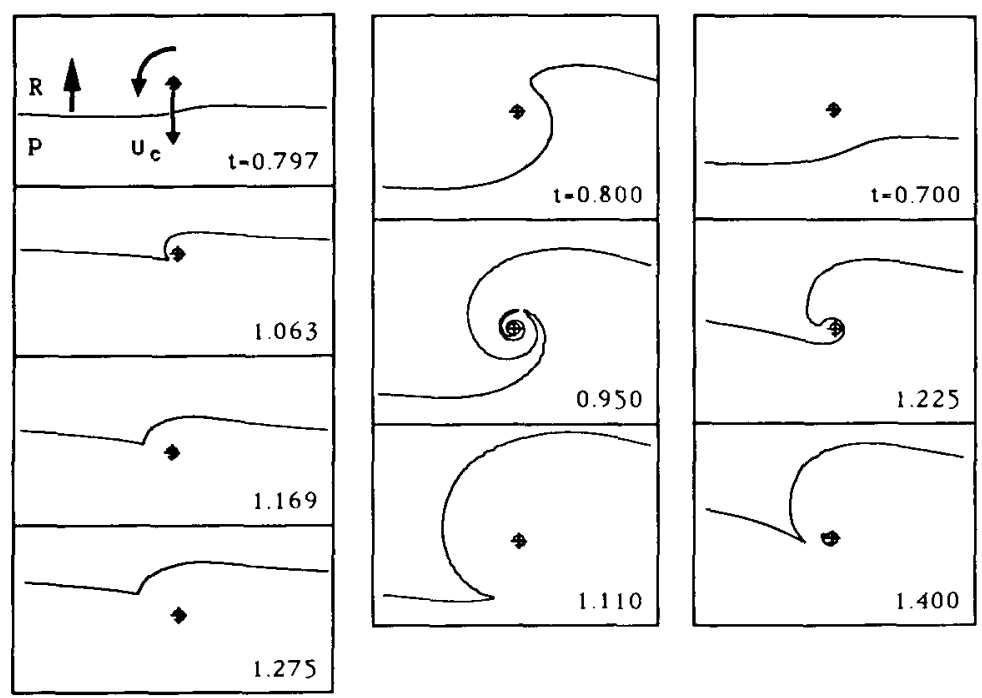
(a) $U_{\theta} / S_{L}=1$
(b) $U_{\theta} / S_{L}=10$
(c) $U_{\theta} / S_{L}=10$
$u_{c} / u_{\theta}=0.063$
$u_{C} / U_{\theta}=0.0$
$u_{C} / u_{\theta}=0.25$

Fig. 9. Simulation of a single vortex convected downward through a premixed flame. $U_{x, \max } / U_{\theta}$ is 1.0 . Time is defined in Fig. 2 . 

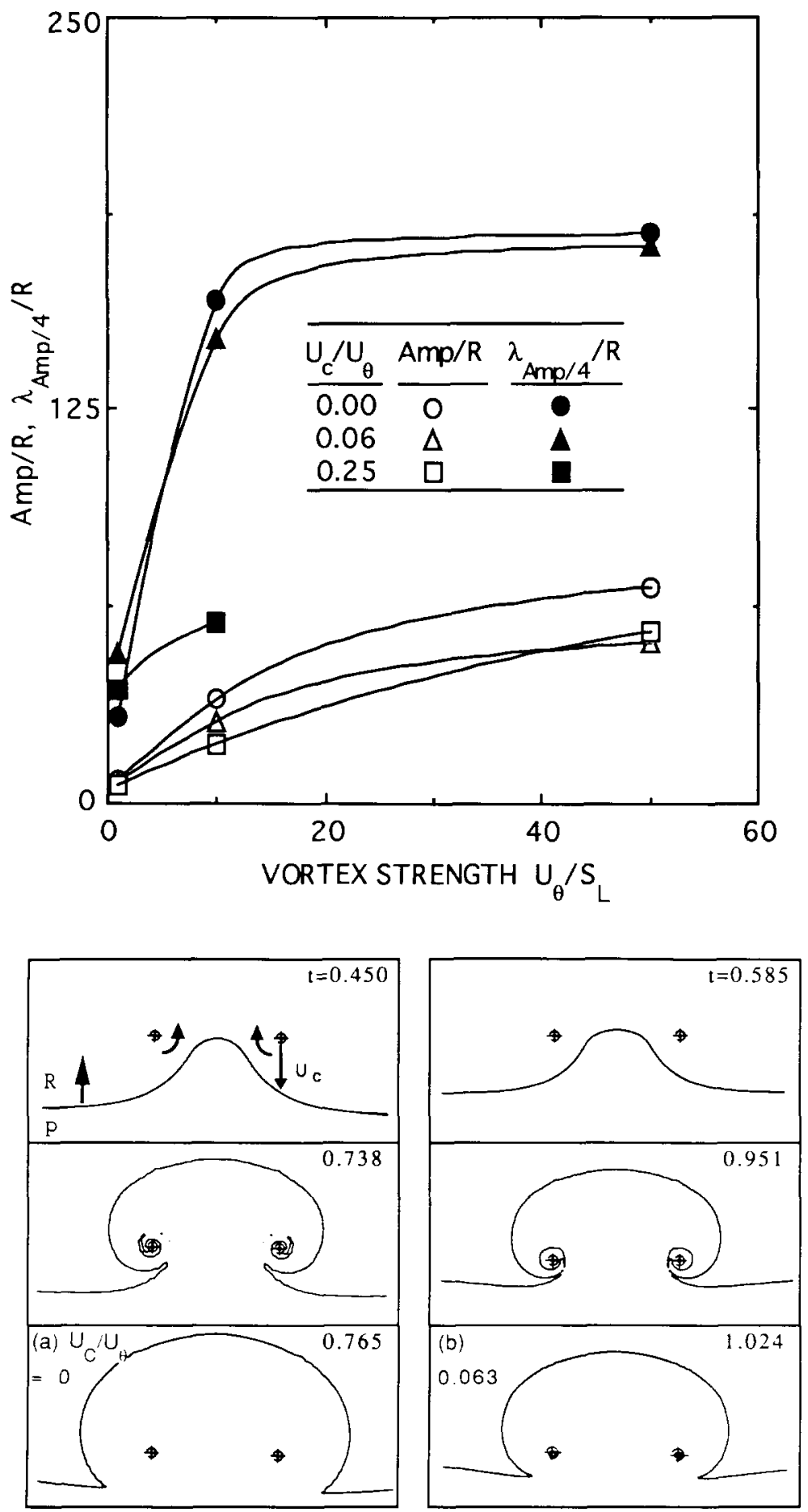

Fig. 11. Flame rollup when vortex velocity is opposite to that of Figs. $1-5 . R / y_{0}$ is 0.08 and $U_{\theta} / S_{L}$ is 10 . Time is defined in Fig. 2.
Fig. 10. Degree of wrinkling of a premixed flame, as characterized by the wrinkle amplitude amp and wrinkle wavelength $\lambda_{\mathrm{Amp} / 4}$. Single vortex case.

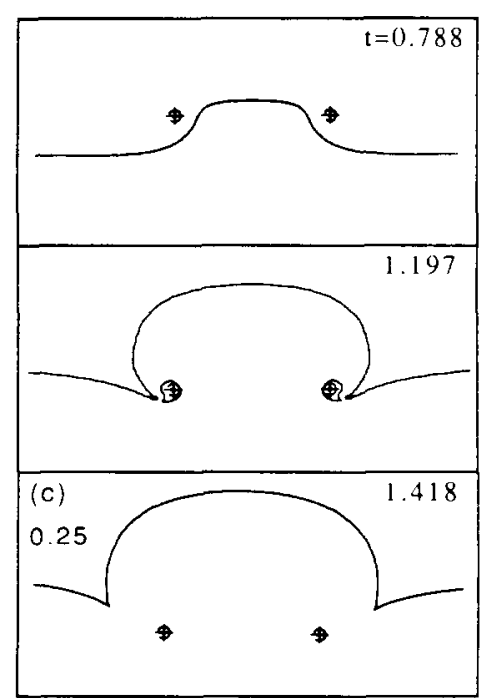


4. Pockets of unburned reactants are predicted to occur, but only for a certain range of convection velocities of the laminar vortex.

This work was aided by a number of useful discussions with Prof. G. M. Faeth concerning the interface simulation and by discussions with $W$. L. Roberts concerning his experiment.

\section{REFERENCES}

1. Chorin, A. J., J. Comp. Phys. 35:1-11 (1980).

2. Peters, N., and Williams, F. A., Twenty-Second Symposium (International) on Combustion, The Combustion Institute, Pittsburgh, 1988, pp.495-503.

3. Chate, H., and Cant, R. S., Combust. Flame, 74:1-8 (1988).

4. Ashurst, W. T., and McMurtry, P. A., Combust. Sci. Technol. 66:17-37 (1989).

5. Rutland, C. J., Ferziger, J. H., and El Tahry, S. H., Twenty-Third Symposium (International) on Combustion, The Combustion Institute, Pittsburgh, 1990, pp.621-627.

6. Laverdant, A., and Candel, S., J. Propul. Power 5:134-143 (1988).

7. Poinsot, T., Veynante, D., and Candel, S., TwentyThird Symposium (International) on Combustion, The Combustion Institute, Pittsburgh, 1990, pp. 613-618.

8. Poinsot, T., Veynante, D., and Candel, S., J. Fluid Mech. 228:561-606 (1991).

9. Roberts, W. L., and Driscoll, J. F., Combust. Flame 87:245-256 (1991). [Also see Roberts, W. L., Driscoll,
J. F., Drake, M. C., and Ratcliffe, J., Twenty-Fourth Symposium (International) on Combustion, The Combustion Institute, Pittsburgh, 1992, in press.]

10. Ashurst, W. T., and Barr, P. K., Combust. Sci. Technol. 34:227-256 (1983).

11. Ghoniem, A. F., and Knio, O. M., Twenty-First Symposium (International) on Combustion, The Combustion Institute, Pittsburgh, 1986, pp. 1313-1320.

12. Wu, M.-S., Kwon, S., Driscoll, J. F., and Faeth, G. M., Combust. Sci. Technol. 83:187-202 (1992).

13. Kwon, S., Wu, M.-S., Driscoll, J. F., and Faeth, G. M., Combust. Flame 88:221-238 (1992).

14. Kerstein, A. R., Ashurst, W. T., and Williams, F. A., Phys. Rev. A 37:2728-2731.

15. Ashurst, W. T., Sivashinsky, G. I., and Yakhot, V., Combust. Sci. Technol. 62:273-284 (1988).

16. Osher, S., and Sethian, J. A., J. Comp. Phys. 79:12-49 (1988).

17. Cantwell, B., and Rott, N., Phys. Fluids 31:3213-3224 (1988).

18. Noh, W., and Woodward, P., Proceedings of the Fifth International Conference on Numerical Methods in Fluid Dynamics (A. I. Vooren and P. J. Zandbergen, Eds.), Springer-Verlag, Berlin, 1976, pp. 330-339.

19. Sethian, J., J. Comp. Phys. 54:425-456 (1984).

20. Driscoll, J. F., and Gulati, A., Combust. Flame 72:131-152 (1988).

21. Wu, M.-S., Kwon, S., Driscoll, J. F., and Faeth, G. M., Combust. Sci. Technol. 78:69-96 (1991).

22. Ashurst, W. T., Kerstein, A. R., Kerr, R. M., and Gibson, C. H., Phys. Fluids 30:2343-2353 (1987).

23. Haworth, D. C., and Poinsot, T. J. (submitted).

Received 29 January 1992; revised 30 June 1992 